\title{
Agent-Assisted Navigation for Virtual Worlds
}

\author{
Fahad Shah, Philip Bell, and Gita Sukthankar \\ University of Central Florida \\ sfahad@cs.ucf.edu, ph.bell@knights.ucf.edu, gitars@eecs.ucf.edu
}

\section{Introduction and Background}

This paper describes the design and training of an agent that helps users navigate in Second Life (SL), a massively multi-player online game environment.SL allows users to create a virtual avatar and explore areas constructed by other users. With the increasing number of places to visit it is difficult for a user to explore all the places.The built-in keyword search mechanism is fairly limited and users typically find new places through personal exploration or tips from their friends.This motivates the need for a recommendation system that can suggest places to visit, personalized with the user's destination preferences.

In this paper, we present a framework for 1) learning a mapping between users' activities and locations in the virtual world and 2) predicting their future destinations from prior travel patterns. By learning a map of the virtual world, our agent can make recommendations about other locations to visit in the virtual world.This paper builds upon our experience from previous implementation of a similar system [1] to include multi-label learning and explicit user requests.

\section{Application}

The basic dataflow for our destination prediction application is summarized in Figure 1 We use the Waikato Environment for Knowledge Analysis (Weka) [2] to learn models of the users' activities in Second Life.For multi-label learning and prediction we used MULAN (Multi-label classification) [3], a software extension to Weka that contains several methods for multi-label classification.

\section{Results and Conclusion}

To evaluate our system, we conducted a small pilot study examining the perceived utility of the navigational agent at assisting users' with searches in the SL virtual world. Additionally we evaluated the classification performance of the supervised learning algorithms used by our navigational agent.

The purpose of user study was to evaluate the performance of the navigational agent by having the agent assist users' with a scavenger hunt task where the users were given a list of objects to find (with and without) using our HUD.The feedback suggested that our agent does not seem to significantly speed the time required to do the scavenger hunt (20 mins) but still users reported a positive user experience while interacting with

Zs. Ruttkay et al. (Eds.): IVA 2009, LNAI 5773, pp. 543 544, 2009.

(C) Springer-Verlag Berlin Heidelberg 2009 


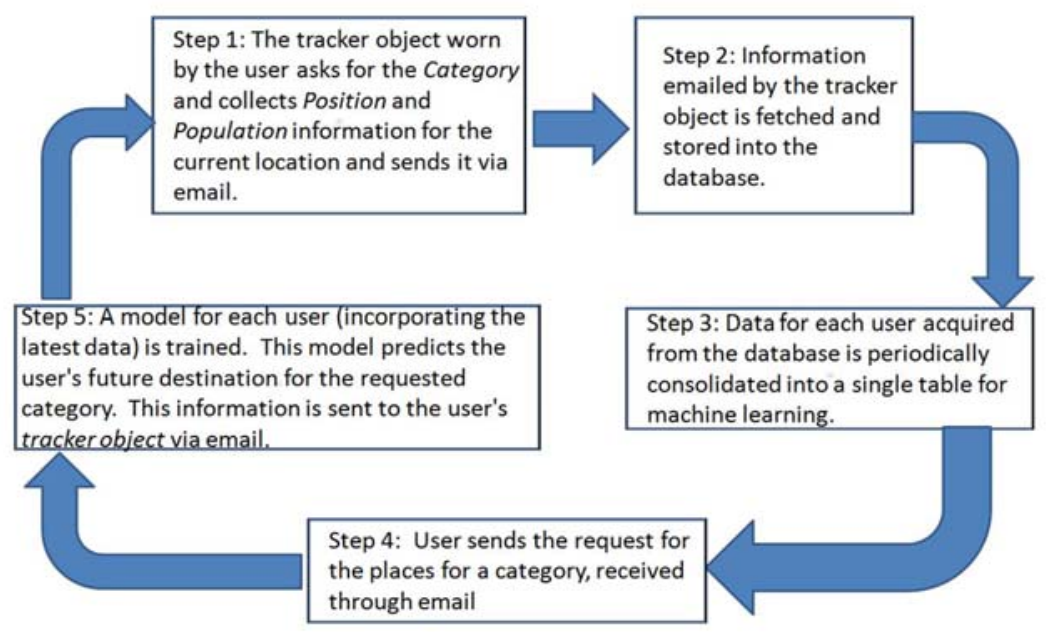

Fig. 1. Application workflow

the agent and said that they liked the idea and plan to use it after the study and also have their friends try it.

The goals of classification performance evaluation task were to: (1) predict the user destination $(x, y, z)$; and (2) predict the region for a given category. The results for destination prediction using the M5P and $\mathrm{KNN}(\mathrm{K}=10$ here) algorithms give a 0.94 or above correlation coefficient for $\mathrm{x}, \mathrm{y}$ an $\mathrm{z}$. This shows excellent correlation between predicted and ground-truth values in all dimensions. We evaluated the prediction for region name using Decision Tree, KNN and Bayes Net with similar classification accuracies of around $76 \%$.Since there are thirty six regions in our database, the baseline (chance) performance on this task is only $0.03 \%$. All of the algorithms perform much better than this baseline, confirming our belief that there is significant spatial structure for user-created attractions in Second Life.

Predictions of the user's activity patterns could be used as a basis for creating peruser experiences and user- targeted advertising. Many people use the internet to create a social presence,this presents an opportunity for researchers to collect rich user data from these interactions to create a more personalized and user-friendly experience.

\section{References}

1. Shah, F., Bell, P., Sukthankar, G.: Identifying user destinations in virtual worlds. In: Florida Artificial Intelligence Research Society (2009)

2. Weka, http://www.cs.waikato.ac.nz/ml/weka/

3. MULAN, http://sourceforge.net/projects/mulan/ 